\title{
ARTícUlo \\ Interacción entre la pesca artesanal y el depredador de alto nivel trófico Lontra felina en Chile
}

\author{
Interaction between artisanal fishery and the top \\ predator Lontra felina in Chile
}

\section{Olivia Córdova ${ }^{1}$ y Jaime R. Rau,**}

\begin{abstract}
'Laboratorio de Biotecnología Ambiental, Escuela de Ingeniería en Bioquímica, Facultad de Ingeniería, Pontificia Universidad Católica de Valparaíso, Av. Brasil 2085, Valparaíso, Chile. ocordova.villarroel@gmail.com

${ }^{2}$ Laboratorio de Ecología, Departamento de Ciencias Biológicas y Biodiversidad, Universidad de Los Lagos, Casilla 933, Osorno, Chile.
\end{abstract} *Autor corresponsal: jrau@ulagos.cl

\begin{abstract}
The sea otter (Lontra felina) lives along the rocky coastline exposed southeast Pacific between Peru $\left(6^{\circ} \mathrm{S}\right)$, Cape Horn, Chile $\left(56^{\circ} \mathrm{S}\right)$ and States island, Argentina. This native carnivore is classified as Vulnerable in the Red Book of Terrestrial Vertebrates of Chile. Between September and November 2004 we assessed their diet through fecal analysis and records of food remnants. We used a quantitative overlap index to test for coincidental similarity between the 2 analyses, and also we applied a survey to fisherfolks at Caleta El Manzano de Pucatrihue, southern Chile, to learn about the perception they have of L. felina impact on local fishing. Based on an analysis of 35 faeces the diet was represented by $60.8 \%$ of crustaceans, $32.4 \%$ of fishes and $6.7 \%$ of shellfishes presences. Of 150 food remnants the prey corresponds to $55.3 \%$ of crustaceans, $44.0 \%$ of molluscs and $0.7 \%$ of birds. The two analyses carried out had a slow similarity $(=0.35 \pm 0.22)$. L. felina preys on resources exploited by artisanal fishing, but fishing gears are not damaged by this marine mammal. A $66.7 \%$ of the fishermen said that otters not affect their fishing, while $30.0 \%$ said the opposite; holding as cause the consumption by L. felina on the same resources exploited by them (e.g., Concholepas concholepas). Although this mammal does not cause significant damage to local fisheries still exists at present, however, illegal hunting of this otter in the study area. The reasons for this action ranging from the sale of their skin until his killing by a simple recreation.
\end{abstract}

Key words: Chungungo, scat analysis, artisanal fishery interaction, Chile

\begin{abstract}
Resumen.- La nutria marina (Lontra felina) habita a lo largo del litoral rocoso expuesto del Pacífico sudeste entre Perú ( $\left.6^{\circ} \mathrm{S}\right)$, Cabo de Hornos, Chile $\left(56^{\circ} \mathrm{S}\right)$ e Isla de Los Estados, Argentina. Este carnívoro nativo está clasificado como especie Vulnerable en el Libro Rojo de los Vertebrados Terrestres de Chile. Entre septiembre y noviembre de 2004 se evaluó su dieta a través de análisis fecal y registro de restos alimenticios. Se utilizó el índice de sobreposición cuantitativo para probar coincidencia entre ambos análisis, también aplicamos una encuesta a los pescadores artesanales de la Caleta El Manzano de Pucatrihue, sur de Chile, para conocer la percepción que tienen de L. felina y su impacto sobre la pesca local. Sobre la base del análisis de 35 fecas los crustáceos representan $60,8 \%$, peces un $32,4 \%$ y moluscos un $6,7 \%$. De 150 restos alimenticios los crustáceos corresponden a 55,3\%, moluscos a un $44,0 \%$ y aves a un $0,7 \%$. Los dos análisis efectuados tuvieron una baja similitud $(=0,35 \pm 0,22)$. L. felina depreda sobre recursos explotados por la pesca artesanal; sin embargo, las artes de pesca no son dañadas por este mamífero marino. Un $66,7 \%$ de los pescadores manifestó no ver afectada su pesca a causa del animal, mientras un 30,0\% afirmó lo contrario; sosteniendo como causa el consumo por parte de L. felina sobre los mismos recursos explotados por ellos (e.g., Concholepas concholepas). Aunque este mamífero no causa daños significativos a la pesca local existe todavía (2003-2004) la caza ilegal de esta nutria en el área de estudio.
\end{abstract}

Palabras clave: Chungungo, análisis fecas, interacción pesca artesanal, Chile

\section{INTRODUCCIÓN}

Lontra felina (Molina, 1782), conocido en Chile comúnmente con los nombres de 'chungungo', 'gato de mar' y 'chinchimén', es un mamífero marino carnívoro (Familia Mustelidae) nativo que habita a lo largo del litoral rocoso expuesto del Pacífico sudeste, entre Perú $\left(6^{\circ} \mathrm{S}\right)$ y el Cabo de Hornos $\left(56^{\circ} \mathrm{S}\right)$ (Castilla \& Bahamondes 1979).
En el Libro Rojo de los Vertebrados Terrestres de Chile (Glade 1993) este mamífero marino es clasificado en la categoría correspondiente de especie vulnerable. De igual manera se encuentra protegido por la Ley Chilena en el Decreto $\mathrm{N}^{\circ} 40$ del 22 de febrero de 1972 y está incluido en la Convención Internacional de Especies Amenazadas, subscrita por el Gobierno de Chile el 16 de septiembre de 1994 (Castilla \& 
Bahamondes 1979), además de ser considerado en peligro y figurar en el Libro Rojo de la Unión Internacional para la Conservación de la Naturaleza (UICN). No existen referencias precisas sobre las causas del por qué L. felina se encuentra catalogada como especie vulnerable, sólo se cuenta con algunos antecedentes como su caza ilegal. En relación con esta práctica diversos autores indican las posibles causas. Según Cabello (1983) serían la falta de vigilancia para el cumplimiento de la ley, además de la existencia de una importante población de pescadores y a la vez 'cazadores' que viven en condiciones precarias y la persistencia de un poder comprador para la venta de sus pieles por parte de los miembros de comunidades costeras. Se considera también la destrucción de hábitat y la interacción antrópica, tanto involucrando la captura del animal así como el aumento de los asentamientos humanos y las actividades económicas realizadas en su hábitat, sensu lato (Medina 1995). Además, por su pequeño tamaño (Larivíere 1998) el animal es capturado, accidentalmente, en redes de pesca utilizadas por pescadores artesanales (Rozzi \& TorresMura 1990). A pesar de la protección legal, tanto nacional como internacional, su caza ilegal continúa, al menos cuando este estudio fue realizado. Las poblaciones chilenas de esta nutria en particular han sido estudiadas en diversos aspectos, que están relacionados con su distribución y abundancia (Cabello 1983, Rozzi \& Torres-Mura 1990, Sielfeld \& Castilla 1999), características y selección de hábitat (Sielfeld 1990, Ebensperger \& Castilla 1991, Medina-Vogel et al. 2006, 2007), dieta y conducta (Castilla \& Bahamondes 1979, Castilla 1982, Ostfeld et al. 1989, Sielfeld 1990, Ebensperger \& Castilla 1991, Medina 1995, Villegas et al. 2007, Badilla \& GeorgeNascimento 2009, Córdova et al. 2009, Mangel et al. 2011), inmovilización química e implante de radio-transmisores (SotoAzat et al. 2006, 2008), sinantropía (Cursach et al. 2012) y filogeografía (Vianna et al. 2010). Algunos de estos estudios se han realizado, esencialmente, con el fin de caracterizar las poblaciones y crear planes estratégicos para la conservación de la especie.

Sielfeld \& Castilla (1999) sugirieron que una de las principales limitantes para el establecimiento de poblaciones o grupos del chungungo son, entre otros, la disponibilidad de alimento. Por otra parte, su dieta en Tacna, Perú, ha sido estudiada por Biffi \& Iannacone (2010), encontrándose un predominio de artrópodos $(59,1 \%)$, seguido por peces $(27,3 \%)$ y moluscos $(13,6 \%)$.

Gran parte de las especies que constituyen la dieta de $L$. felina tienen importancia comercial para las caletas artesanales (especies de Cancer spp., peces de la zona submareal, moluscos como los 'locos', Concholepas concholepas). En este contexto, resulta de interés preguntarse si L. felina es percibida por los pescadores artesanales como una amenaza que causa impacto en sus actividades de pesca local.

El objetivo del presente estudio fue obtener datos de la percepción del daño aparente de chungungos sobre los recursos pesqueros de la comunidad de pescadores artesanales (i.e., etno-mastozoología) de la Caleta de Pucatrihue, Osorno, sur de Chile. Secundariamente, se entrega información sobre su dieta en el sur de Chile empleando dos métodos complementarios de análisis.

\section{Materiales Y MÉTODOS}

\section{Sitio de ESTUdio}

El presente estudio se realizó en el islote Huenteyao, ubicado en la costa de Pucatrihue frente al Area de Manejo de Recursos Bentónicos (AMERB) El Manzano de Pucatrihue, Región de los Lagos, $\left(40^{\circ} 28^{\prime} \mathrm{S} ; 73^{\circ} 43^{\prime} \mathrm{W}\right)$; esta localidad se encuentra situada a unos $65 \mathrm{~km}$ al Oeste de la ciudad de Osorno, sur de Chile. El islote mide aproximadamente $510 \mathrm{~m}$ de longitud (P. Vergara, com. pers.) y se encuentra rodeado por un cinturón de macroalgas café (Durvillea antartica). En tierra presenta densa vegetación encontrándose especies como Plantago lanceolata, Rubus ulmifolius y Greigia sphacelata. En este lugar habitan principalmente colonias de aves tales como Coragyps atratus, Cathartes aura, Pelecanus thagus, Haematopus palliatus, Spheniscus magellanicus y Larus dominicanus (Cursach et al. 2009).

Se aplicó una encuesta a 26 de los 50 pescadores artesanales de la caleta El Manzano de Pucatrihue inscritos en el sindicato homónimo, con el fin de identificar las especies explotadas, conocer las artes de pescas empleadas en la captura de las especies y la influencia que tiene L. felina en su pesca local. Esta se estructuró como un cuestionario conformado por las siguientes 4 preguntas cerradas: ¿Cuántos chungungos ha cazado? (1 a 2, 2 a 4, 4 a 8); ¿Cree que su pesca se ve afectada por el chungungo? (Sí, no, no responde); ¿Ve ahora más o menos chungungos que hace 10 años atrás? (Más, menos, igual, no responde); ¿De qué manera le afecta el chungungo en su pesca? (Alimentándose de los recursos explotados, no responde), (más detalles en Cursach et al. 2012). Existe un total de 66 socios inscritos en el sindicato de la caleta El Manzano de Pucatrihue de los cuales 32 son buzos, 2 ayudantes de buzo, 16 pescadores, 12 armadores y 15 algueros (Vergara 2004) $)^{1}$. Cincuenta son los socios que tienen participación activa.

\footnotetext{
${ }^{1}$ Vergara PA. 2004. Informe de seguimiento N² del Área de Manejo El Manzano de Pucatrihue, X Región. Asesorías y Capacitación en Acuicultura Limitada, Departamento de Áreas de Manejo y Pesquerías. Informe mimeografiado, 23 pp.
} 
Adicionalmente, se determinaron mediante dos metodologías complementarias los hábitos alimenticios del chungungo para conocer la dieta local del animal. Para esto se colectaron muestras de sus fecas ( $n=35$, incluidas en el análisis de 106 muestras efectuado por Córdova et al. 2009) y se registraron sus restos de alimentos en comederos quincenalmente entre noviembre de 2003 a septiembre de 2004 (este estudio). Los restos de alimentos fueron recolectados desde un comedero (roca plana de $11 \mathrm{~m}$ de largo máximo y 13,6 m de ancho máximo), caracterizado por presentar gran cantidad de restos de crustáceos como quelas y caparazones y conchas de moluscos.

Las muestras de fecas y de restos de alimentos fueron analizadas utilizando el protocolo propuesto por Sielfeld (1990). Se conservaron las fecas en envolturas de papel de diario para luego secarlas en estufa a $90^{\circ}$ grados durante $12 \mathrm{~h}$. Se separaron los restos de alimento por categorías de presas para luego ser identificados al máximo nivel de resolución taxonómica posible. El trabajo de laboratorio consistió en la disgregación de las fecas ya secas y la posterior identificación de los restos de las presas encontradas con la ayuda de claves taxonómicas y catálogos de referencia (Webb 1976, Retamal 1981, Falabella et al. 1995).

Se probó la coincidencia entre los dos métodos utilizados (análisis fecal e identificación de restos de alimento), para establecer la similitud de la dieta, con el índice cuantitativo de Czechanowsky (sensu N. Gotelli) comparándolo contra un modelo nulo (i.e., la similitud es 0 ) con el programa computacional ECOSIM que emplea reasignaciones estocásticas ('Boostrap') e iteraciones de matrices generadas al azar. Para ello utilizamos una matriz de dos columnas (ambos métodos) y 22 filas de los ítemes-presas identificados en ambos casos, el algoritmo de aleatorización RA3 ("niche breadth retained and zero states reshuffled') y un uso equiprobable de los recursos (http://www.uvm.edu/ ngotelli/EcoSim/ EcoSim.html).

\section{Resultados}

\section{ENCUESTA}

Se obtuvieron los siguientes datos de las respuestas de la encuesta realizada a los pescadores artesanales: el porcentaje de pescadores artesanales que comercializan peces y mariscos alcanza el 96,3\%, siendo la caleta el Manzano de Pucatrihue y la ciudad de Osorno los lugares para su comercialización. El $100 \%$ de los pescadores encuestados conoce al chungungo con el nombre de 'gato de mar' observándolo durante todo el año en la costa rocosa y el islote. El 100\% de los pescadores responde que, basados en observación directa del animal, este se alimentaría de jaibas y peces que son las principales especies consumidas por L. felina (Tabla 1). El 70,3\% creen que en la actualidad existen más chungungos que hace diez años atrás, mientras que un $7,4 \%$ cree que hay menos y un $11,1 \%$ cree que las poblaciones no han ni aumentado ni disminuido. Las posibles causas que explicarían esta disminución, según los propios pescadores, serían la caza ilegal, la falta de alimento y la migración del animal. Dieciséis pescadores $(59,2 \%)$ sostienen que nunca han cazado chungungos mientras que $6(22,2 \%)$ afirman haber cazado a lo menos 2 chungungos en menos de un año, $5(18,5 \%)$ pescadores no quisieron responder a la pregunta sobre la caza ilegal del animal. Con respecto al posible daño que provocaría L. felina en la pesca y en las artes de pesca, la mayoría de los pescadores $(66,7 \%)$ considera que no es afectado por la interacción de la nutria en sus productos capturados mientras que aproximadamente un 30,0\% afirma lo contrario, sosteniendo que la principal causa es que consume los mismos recursos explotados por ellos.

Tabla 1. Principales recursos explotados por los pescadores artesanales de la caleta El M anzano de Pucatrihue. Los datos fueron obtenidos sobre la base de las respuestas de los pescadores y al Estudio de la Situación Base del Área (E.S.B.A.) realizado en dicha localidad por Vergara (2004) 1 / Main resources exploited by artisanal fishermen in El Manzano of Pucatrihue study area. These data were obtained on the basis of responses from fishermen and to Study of the Basis Situation (ESBA) made in that locality by Vergara $(2004)^{1}$

\begin{tabular}{|c|c|c|}
\hline & Nombre común & Nombre científico \\
\hline \multirow[t]{10}{*}{ Peces } & Corvina & Cilus gilberti \\
\hline & Róbalo & Eleginops maclovinus \\
\hline & Pejerrey & Odontesthes regia \\
\hline & Mantaralla & Raja flavirostris \\
\hline & Congrio & Genypterus chilensis \\
\hline & Jurel & Trachurus symmetricus \\
\hline & Pejegallo & Callorhinchus callorynchus \\
\hline & Sierra & Thyrsites atun \\
\hline & Rollizo & Pinguipes chilensis \\
\hline & Merluza & Merluccius gayi \\
\hline \multirow[t]{9}{*}{ Moluscos } & Lapa & Fissurella spp. \\
\hline & Loco & Concholepas concholepas \\
\hline & Picoroco & Austromegabalanus psittacus \\
\hline & Choro zapato & Choromytilus chorus \\
\hline & Culengue & Gari solida \\
\hline & Choritos & Mytilus chilensis \\
\hline & Chorito maico & Perumytilus purpuratus \\
\hline & Caracol negro & Tegula atra \\
\hline & Caracol palo palo & Argobuccinum pustulosum \\
\hline \multirow[t]{2}{*}{ Crustáceos } & Jaiba peluda & Cancer setosus \\
\hline & Jaiba mora & Homalaspis plana \\
\hline Equinodermos & Erizo & Loxechinus albus \\
\hline Tunicados & Piure & Pyura chilensis \\
\hline
\end{tabular}


Tabla 2. Frecuencia de ocurrencia y frecuencia relativa (\%) de presas de especies y familias en 35 fecas de $L$. felina colectadas en la localidad de Pucatrihue, sur de Chile / Occurrence frequency and relative frequency (\%) of prey species and families in 35 feces of L. felina collected in the locality of Pucatrihue, southern Chile

\begin{tabular}{lcc}
\hline Especie o Familia & Ocurrencia & $\begin{array}{c}\text { Frecuencia } \\
\text { relativa (\%) }\end{array}$ \\
\hline Crustáceos & & \\
Homalaspis plana & 11 & 34,3 \\
Taliepus dentatus & 6 & 18,7 \\
Paraxanthus barbiger & 8 & 25,0 \\
Cancer setosus & 7 & 21,8 \\
Cancer spp. & 6 & 18,7 \\
Isopoda & 4 & 5,4 \\
Indeterminados & 3 & 9,3 \\
Total & 45 & 60,8 \\
Peces & & \\
Percidae & 5 & 23,8 \\
Eleginops maclovinus & 4 & 19,0 \\
Sicyases sanguineus & 2 & 9,5 \\
Genypterus maculatus & 1 & 4,7 \\
Pinguipes chilensis & 1 & 4,7 \\
Merluccius gayi & 1 & 4,7 \\
Prolatilus jugularis & 1 & 4,7 \\
Cilus gilberti & 2 & 9,5 \\
Bovichthys chilensis & 2 & 9,5 \\
Sebastes oculatus & 1 & 4,7 \\
Indeterminados & 4 & 19,0 \\
Total & 24 & 32,4 \\
Moluscos & & \\
Fissurella spp. & 5 & 6,7 \\
\hline
\end{tabular}

\section{ANÁlisis FECAL}

Se reconocieron 3 categorías de presas: crustáceos $(60,8 \%)$, peces $(32,4 \%)$ y moluscos $(6,7 \%)$. Dentro de la primera categoría fueron identificadas 5 especies de crustáceos. Homalaspis plana $(34,3 \%)$ y Paraxanthus barbiger (25\%) fueron las especies de crustáceos con mayor frecuencia de ocurrencia (Tabla 2).

A diferencia de la categoría de presa anterior, entre los peces fue posible identificar a 9 especies que constituyen la dieta del chungungo. Representantes del orden Perciformes con un $23,8 \%$, seguidos por Eleginops maclovinus con $19 \%$, presentaron las mayores frecuencias de ocurrencia. El grupo moluscos sólo fue representado por el género Fissurella, no superando el $10 \%$.
Tabla 3. Frecuencia de ocurrencia y frecuencia relativa (\%) de las categorías de presas de $\mathbf{1 5 0}$ restos de alimentos recolectados de un comedero ubicado en la localidad de Pucatrihue, $X$ Región, sur de Chile / Occurrence frequency and relative frequency (\%) of the categories of prey collected 150 food remnants of a trough located in Pucatrihue, $X$ Region, southern Chile

\begin{tabular}{lcc}
\hline \multicolumn{1}{c}{ Especie o Familia } & \multicolumn{2}{c}{ Frecuencia de ocurrencia } \\
& absoluta & relativa (\%) \\
\hline Crustáceos & & \\
Homalaspis plana & 38 & 25,3 \\
Cancer setosus & 22 & 14,7 \\
Paraxanthus barbiger & 15 & 10,0 \\
Taliepus dentatus & 8 & 5,3 \\
Total & 83 & 55,3 \\
Moluscos & & \\
Concholepas concholepas & 40 & 26,7 \\
Fissurella spp. & 12 & 8,0 \\
Tegula atra & 7 & 4,7 \\
Scurria scurria & 5 & 3,3 \\
Venus antiqua & 2 & 1,4 \\
Total & 66 & 44 \\
Aves & 1 & 0,7 \\
\hline
\end{tabular}

\section{Restos de ALIMENTo EN COMEDEROS}

Fueron identificadas 5 especies de crustáceos, siendo nuevamente el grupo de presas que alcanzó la mayor frecuencia de ocurrencia $(55,3 \%)$. H. plana $(25,3 \%)$ y Cancer setosus $(14,2 \%)$ fueron las 2 especies con mayor frecuencia de ocurrencia en la dieta (Tabla 3). La categoría de presa moluscos aumentó con relación al análisis fecal alcanzando un $44 \%$ de los restos de alimentos encontrados, estando constituidos por el molusco de importancia comercial Concholepas concholepas (26,7\%) y Fissurella spp. (8,0\%). Es destacable que la mayoría de las especies de moluscos encontrados en los restos de alimentos no fueron halladas en las fecas (así como los peces sí lo fueron en ellas y no en los comederos).

En el caso del método de análisis fecal, no fue posible identificar moluscos ya que el chungungo sólo consume y digiere las partes blandas del animal, las cuales sí son posibles de identificar con el método basado en restos de alimentos ya que L. felina deja en los comederos sólo los restos que no puede consumir como por ejemplo conchas de moluscos (e.g., C. concholepas). Esto se repite para otras categorías de presas (aves).

Fue posible observar también restos de alimentos en oquedades de grandes rocas, sin embargo, la profundidad de estas cuevas nos impidió obtener restos de alimentos de estos lugares. 
El índice de similitud cuantitativo para medir la sobreposición entre los dos métodos empleados indicó que existió una baja similitud igual a $0,35 \pm 0,22$ (media aritmética \pm varianza) y ésta difirió estadísticamente y en forma altamente significativa $(P<0,00001)$ de lo esperado para un modelo nulo (i.e., que fuese igual a 0). Por tanto, se estima entonces, que ambos métodos deben utilizarse siempre de manera complementaria para caracterizar la dieta del chungungo.

\section{Discusión}

En este estudio la mayoría de los pescadores artesanales de la caleta no tiene una actitud negativa frente a $L$. felina, esto porque el $66 \%$ de los encuestados perciben que su pesca no se ve afectada, de ninguna manera, por L. felina en la Caleta El Manzano de Pucatrihue. No obstante, y como consecuencia que su hábitat natural ha sido ocupado por los pescadores artesanales, L. felina depreda sobre recursos económicamente explotados por ellos en la caleta El Manzano de Pucatrihue. Esto es confirmado con el análisis de la dieta de L. felina la que incluye principalmente a las categorías de presas crustáceos, peces y moluscos. A través de la información disponible de la conducta alimenticia del mustélido es posible confirmar, por la similitud de los resultados registrados, que L. felina es principalmente consumidor de especies invertebradas tales como crustáceos que en promedio constituyen el 58,0\% de su dieta. Estos resultados concuerdan con los obtenidos por Medina-Vogel et al. (2004) y Córdova et al. (2009) para estudios realizados en el sur de Chile y difieren de los efectuados por Biffi \& Iannacone (2010) en Tacna, Perú, quienes encontraron un predominio de artrópodos $(59,1 \%)$, seguido por peces $(27,3 \%)$ y moluscos $(13,6 \%)$. En cuanto a los métodos utilizados en la determinación de la dieta de L. felina estos tienen grados de similitud baja, con lo que se espera que sólo las categorías de presas comunes encontradas tanto en el análisis fecal como en la identificación de restos de alimentos sean similares en un porcentaje inferior al $65 \%$ (i.e., 100$35,1 \%$ ). Con este reducido grado de similitud se demuestra que ambos métodos se deben siempre usar y considerar como complementarios para determinar la dieta del chungungo.

Aunque existe una sobreposición en el uso del recurso entre chungungos y pescadores artesanales, ésta no sería causal de daños, porque el volumen de alimento que consume L. felina no sería significativo en comparación con el volumen de peces capturados por los pescadores (O. Córdova, obs. pers.), por lo demás L. felina captura sus presas en el mar únicamente en un radio de 100-150 m (Castilla 1982) por lo que no representa una amenaza para la pesca costera comercial que se efectúa mar adentro. Sin embargo, L. felina sí representaría un aparente conflicto para los pescadores artesanales y sus familias que únicamente mariscan en los alrededores de la costa rocosa y en el islote Huenteyao, hábitat del chungungo en el área de estudio (e.g., al alimentarse de C. concholepas).

Las artes de pesca no serían dañadas por parte de L. felina, ya que están confeccionadas de materiales difíciles de destrozar; sin embargo, ello no impide que las nutrias queden enredadas en redes generando su captura incidental, caso reportado por Rozzi \&Torres-Mura (1990).

En la localidad de Pucatrihue durante este estudio existió caza ilegal de la especie. Las principales causas que parecen motivarla serían para evitar el consumo de parte de L. felina sobre los organismos de importancia económica extraídos por los pescadores y la venta de su piel por necesidad económica, o bien su matanza por simple diversión o recreación, esto porque los propios pescadores artesanales de la caleta en conversaciones informales nos señalaron que a los chungungos les lanzan piedras y palos para provocar su muerte. Esta conducta la realizan principalmente pescadores que en absoluto tienen un entendimiento de la importancia de conservar a esta especie. Por tanto, en este último caso es necesario llevar a cabo propuestas encaminadas a reducir esta mortalidad y que en paralelo no afecte al éxito de captura de las presas recolectadas por los pescadores artesanales. En efecto, la mortalidad de L. felina, es relativamente baja, si consideramos que sólo 6 pescadores reconocen haber matado de 1 a 2 chungungos en los últimos años, sin embargo, no es posible cuantificar el daño que causan los pescadores artesanales a $L$. felina ya que en la zona no existen datos de densidad del mustélido.

La caza de L. felina en Chile es ilegal por lo que los pescadores artesanales que entrevistamos podrían ocultar información con respecto a la muerte del animal. Sin embargo, la posesión de pieles de una especie que está protegida, por parte de algunos pescadores, refleja que no existe una real fiscalización al cumplimiento de la ley. La matanza del animal es importante desde el punto de vista de la conservación de la especie ya que limita las posibilidades de eventos de reproducción de los chungungos. En la última década, la situación descrita ha cambiado favorablemente para la especie; sin embargo, las amenazas actuales son el ataque por perros y envenenamiento con venenos para roedores (Cursach et al. 2012).

En cuanto a la conservación de su hábitat, tiene poco sentido la protección de la especie si paralelamente su hábitat se está continuamente degradando. Una herramienta de protección que ofrece eficaces resultados son los Parques Marinos (véase

${ }^{2}$ Santibáñez A. 2005. Uso diferencial del ambiente acuático y terrestre por la nutria marina Lontra felina (Molina, 1782) (Carnivora: Mustelidae), durante las estaciones invierno-primavera, en la costa rocosa de Quintay, $\mathrm{V}$ Región de Valparaíso, Chile. Tesis de pregrado, Universidad Austral de Chile, Valdivia, Chile, 25 pp. 
revisión en Fernández \& Castilla 2005). Santibáñez (2005)² señala que la utilización del medio terrestre (zona del litoral) es preferida por L. felina sobre el medio acuático en un $80 \%$, por lo que propone utilizar los Parques Marinos como herramienta de conservación, ya que las Reservas Marinas están más abocadas a la sustentabilidad de recursos productivos, con posibilidades de extracción de recursos tanto bentónicos como pelágicos, además de realizarse allí otras actividades (Morales \& Ponce 1997).

En el mismo contexto dentro de los Parques Marinos, según la Ley General de Pesca y Acuicultura (LGPA), quedan prohibidas actividades humanas que no estén relacionadas con las autorizadas en el marco de observación, investigación o estudios científicos (Morales \& Ponce 1997) por lo que ésta pareciera ser la mejor opción de protección para las poblaciones del chungungo.

\section{Agradecimientos}

Se agradece al M. Cs. Pedro Vergara por compartir valiosa información sobre la AMERB 'El Manzano de Pucatrihue' y a la Dirección de Investigación de la Universidad de Los Lagos por financiar los costos de esta publicación.

\section{LITERATURA CITADA}

Badilla M \& M George-Nascimento. 2009. Conducta diurna del chungungo Lontra felina (Molina, 1782) en dos localidades de la costa de Talcahuano, Chile: ¿Efectos de la exposición al oleaje? Revista de Biología Marina y Oceanografía 44: 409-415.

Biffi D \& J Iannacone. 2010. Variabilidad trófica de Lontra felina (Molina 1782) (Carnivora: Mustelidae) en dos poblaciones de Tacna (Perú) entre agosto y diciembre de 2006. Mastozoología Neotropical 17: 11-17.

Cabello C. 1983. La nutria de mar en la isla de Chiloé. Boletín Técnico, CONAF 6: 1-37.

Castilla JC. 1982. Nuevas observaciones sobre conducta, ecología y densidad de Lutra felina (Molina 1782) (Carnivora: Mustelidae) en Chile. Museo Nacional de Historia Natural, Publicación Ocasional 38: 197-206.

Castilla JC \& I Bahamondes. 1979. Observaciones conductuales y ecológicas sobre Lutra felina (Molina) 1782 (Carnivora: Mustelidae) en las zonas central y centro-norte de Chile. Archivos de Biología y Medicina Experimentales 12:119-132.

Córdova O, JR Rau, CG Suazo \& A Arriagada. 2009. Estudio comparativo de la ecología alimentaria del depredador de alto nivel trófico Lontra felina (Molina, 1782) (Carnivora: Mustelidae) en Chile. Revista de Biología Marina y Oceanografía 44: 429-438.
Cursach J, J Vilugrón, C Tobar, J Ojeda, J Rau, C Oyarzún \& O Soto. 2009. Nuevos sitios de nidificación para cuatro especies de aves marinas en la provincia de Osorno, centrosur de Chile. Boletín Chileno de Ornitología 15: 17-22.

Cursach JA, JR Rau, F Ther, J Vilugrón \& CN Tobar. 2012. Sinantropía y conservación marina: el caso del chungungo Lontra felina en el sur de Chile. Revista de Biología Marina y Oceanografía 47: 593-597.

Ebensperger LA \& JC Castilla. 1991. Conducta y densidad poblacional de Lutra felina en Isla Pan de Azúcar (III Región, Chile). Medio Ambiente 11:79-83.

Falabella F, R Meléndez \& ML Vargas. 1995. Claves osteológicas para peces de Chile central, un enfoque arqueológico, 208 pp. Editorial Artegrama, Santiago.

Fernández M \& JC Castilla. 2005. Marine conservation in Chile: historical perspective, lessons, and challenges. Conservation Biology 19: 1752-1762.

Glade A. 1993. Libro rojo de los vertebrados terrestres de Chile. CONAF, 68 pp. Ministerio de Agricultura, Santiago.

Larivíere S. 1998. Lontra felina. Mammalian Species. American Society of Mammologists 75: 1-5.

Mangel JC, T Whitty, G Medina-Vogel, J Alfaro-Shigueto, C Cáceres \& BJ Godley. 2011. Latitudinal variation in diet and patterns of human interaction in the marine otter. Marine Mammal Science 27: E14-E25.

Medina G. 1995. Feeding habits of marine otter (Lutra felina) in southern Chile. Proceedings of the International Otter Colloquium 6: 65-68.

Medina-Vogel G, C Delgado, R Alvarez \& JL Bartheld. 2004. Feeding ecology of the marine otter (Lutra felina) in a rocky seashore of the south of Chile. Marine Mammal Science 20: 134-144.

Medina-Vogel G, JL Bartheld, R Alvarez-Pacheco \& C Delgado-Rodríguez. 2006. Population assessment and habitat use by marine otter Lontra felina in southern Chile. Wildlife Biology 12: 191-199.

Medina-Vogel G, F Boher, G Flores, A Santibañez \& C Soto-Azat. 2007. Spacing behavior of marine otters (Lontra felina) in relation to land refuges and fishery waste in central Chile. Journal of Mammalogy 88: 487-494.

Morales C \& F Ponce. 1997. Parques marinos y reservas marinas en la Ley General de Pesca y Acuicultura: desafíos para su establecimiento y aplicación. Estudios Oceanológicos, Chile 16: 19-26.

Ostfeld RS, L Ebensperger, L Klostermand \& JC Castilla. 1989. Foraging, activity budget, and social behavior of the South American marine otter Lutra felina (Molina, 1782). National Geographic Research 5: 422-438.

Retamal MA. 1981. Catálogo ilustrado de los crustáceos decápodos de Chile. Gayana Zoología, Chile 44: 1-110. 
Rozzi R \& JR Torres-Mura. 1990. Observaciones del chungungo (Lutra felina) al sur de la isla Grande de Chiloé: antecedentes para su conservación. Medio Ambiente, Chile 11:24-28.

Sielfeld WK. 1990. Dieta del chungungo (Lutra felina, Molina, 1782) (Mustelidae, Carnivora) en Chile Austral. Revista de Investigaciones Científicas y Tecnológicas, Serie Ciencias de Mar, Chile 1: 23-29.

Sielfeld W \& JC Castilla. 1999. Estado de conservación y conocimiento de las nutrias en Chile. Estudios Oceanológicos, Chile 18: 69-79.

Soto-Azat C, F Boher, M Fabry, P Pascual \& G MedinaVogel. 2008. Surgical implantation of intra-abdominal radiotransmitters in marine otters (Lontra felina) in central Chile. Journal of Wildlife Diseases 44: 979-982.
Soto-Azat C, F Boher, G Flores, E Mora, A Santibañez \& G Medina-Vogel. 2006. Reversible anesthesia in wild marine otters (Lontra felina) using ketamine and medetomidine. Journal of Zoo and Wildlife Medicine 37: 535538.

Vianna JA, P Ayerdi, G Medina-Vogel, JC Mangel, H Zeballos, M Apablaza \& S Faugeron. 2010. Phylogeography of the marine otter (Lontra felina): historical and contemporary factors determining its distribution. Journal of Heredity 101: 676689.

Villegas MJ, A A ron \& LA Ebensperger. 2007. The influence of wave exposure on the foraging activity of marine otter, Lontra felina (Molina, 1782) (Carnivora: Mustelidae) in northern Chile. Journal of Ethology 25: 281-286.

Webb JB. 1976. Otter spraint analysis, 15 pp. Occasional Publication of the Mammal Society, Mammal Society, London. 